\title{
Association between coronary artery atherosclerosis and plasma glucose levels assessed by dual-source computed tomography
}

\author{
Yi Yu' ${ }^{1}$, Zhiwen Zhou ${ }^{2}$, Kun Sun ${ }^{2}$, Lili Xi ${ }^{1}$, Lina Zhang ${ }^{3}$, Lingwei Yu ${ }^{4}$, Jing Wang ${ }^{2}$, Jiayi Zheng ${ }^{2}$, Ming $^{4}$ \\ Ding $^{4}$
}

${ }^{1}$ Department of Ultrasound, ${ }^{2}$ Department of Cardiology, Xinhua Hospital Affiliated with the School of Medicine, Shanghai Jiao Tong University, Shanghai 200092, China; ${ }^{3}$ Department of Biostatistics, School of Medicine, Shanghai Jiao Tong University, Shanghai 200025, China; ${ }^{4}$ Department of Radiology, Xinhua Hospital Affiliated with the School of Medicine, Shanghai Jiao Tong University, Shanghai 200092, China

Contributions: (I) Conception and design: Y Yu; (II) Administrative support: K Sun; (III) Provision of study materials or patients: L Yu, L Xi; (IV) Collection and assembly of data: J Wang, J Zheng, M Ding; (V) Data analysis and interpretation: Z Zhou, L Zhang; (VI) Manuscript writing: All authors; (VII) Final approval of manuscript: All authors.

Correspondence to: Yi Yu, MD. Department of Ultrasound, Xinhua Hospital Affiliated with the School of Medicine, Shanghai Jiao Tong University, 1665 Kongjiang Road, Shanghai 200092, China. Email: yuyi20122012@163.com; Kun Sun, MD, PhD. Department of Cardiology, Xinhua Hospital Affiliated with the School of Medicine, Shanghai Jiao Tong University, 1665 Kongjiang Road, Shanghai 200092, China.

Email: Drsunkun@xinhuamed.com.cn.

Background: To assess the association between coronary artery atherosclerosis (CAA) and plasma glucose parameters in a randomly selected cohort of asymptomatic, community-dwelling, Chinese adults by dualsource computed tomography (DSCT).

Methods: We randomly selected participants and classified them into three groups based on their plasma glucose levels: normal glucose regulation (NGR), pre-diabetes, and diabetes mellitus (DM). The participants underwent DSCT, and those identified with CAA were divided into four groups according to the severity of their coronary artery stenosis. We analyzed the composition of plaques in all coronary artery segments according to the American Heart Association's (AHA) guidelines. We compared the severity of coronary artery stenosis and the plaque composition with plasma glucose parameters among participants.

Results: Out of a total of 335 participants, 118 were found to have CAA. The prevalence of CAA was highest (P value for trend $=0.031$ ) in the diabetic group (67.7\%) followed by the pre-diabetic group (35.1\%) then the NGR group (27.7\%). Both calcified and mixed plaques were found in the coronary arteries of the diabetic group while mixed and non-calcified plaques predominated in the pre-diabetic and the NGR groups. When data from all subjects with CAA were analyzed, blood glucose parameters, fasting plasma glucose (FPG), 2-hr postprandial plasma glucose (PPG), and hemoglobin A1c (HbA1c), exhibited a positive correlation with the severity of coronary stenosis $(\mathrm{P}<0.05)$. Multivariable logistic regression models indicated a significantly higher risk of CAA among the diabetic patients. Triglyceride levels were positively correlated with the blood glucose parameters among the three groups while LDL-C was elevated in the DM group but not in the pre-diabetic group compared to the NGR group.

Conclusions: The severity of CAA exhibited a direct correlation with the blood glucose parameters, FPG, PPG, and HbA1c. DSCT can accurately detect the presence and distribution of CAA in asymptomatic, community-dwelling subjects. DSCT is a useful screening tool for coronary artery disease (CAD).

Keywords: Diabetes mellitus (DM); coronary artery atherosclerosis (CAA); dual-source computed tomography (DSCT); plasma glucose parameters; CAA prevention

Submitted Mar 20, 2018. Accepted for publication Sep 28, 2018.

doi: $10.21037 /$ jtd.2018.10.62

View this article at: http://dx.doi.org/10.21037/jtd.2018.10.62

(c) Journal of Thoracic Disease. All rights reserved. 


\section{Introduction}

Patients with diabetes mellitus (DM) are at an increased risk for the development of coronary artery disease (CAD) $(1,2)$, and CAD is the principal cause of morbidity and mortality in diabetic adults $(3,4)$. The study of Rivera et al. (5) demonstrated a higher prevalence of CAD in asymptomatic patients with diabetes than in non-diabetic subjects using cardiac computed tomography angiography (CTA). Using multi-detector CTA, Yang et al. (6) showed an association between hemoglobin A1c (HbA1c) and subclinical coronary artery atherosclerosis (CAA) in patients with type 2 diabetes (T2DM). They suggested that long-term stabilization of blood glucose levels could be important in preventing the progression of subclinical CAA during the early phase of T2DM (6).

Most previous studies of cardiovascular risk in diabetes were performed on diabetic patients with known or suspected CAD. However, there are limited data on CAA in community-dwelling, asymptomatic subjects concerning its association with plasma glucose levels. Furthermore, there is no consensus regarding testing for the presence of CAD in asymptomatic patients with diabetes (7). The latter underscores the need to develop practical approaches amenable to routine, repetitive screening for detecting CAD at an early stage when the success of treatment would be expected to increase.

Dual-source computed tomography (DSCT) is an emerging noninvasive technology capable of identifying the presence of CAD and quantifying its severity (8). It is a reliable alternative to invasive coronary angiography (9). It also has the capability of accurately determining plaque composition and the extent of luminal narrowing for the diagnosis and treatment of obstructive CAD (8,10-13). Thus, DSCT is a potentially valuable tool for the identification of asymptotic subjects at high risk for subsequent, adverse cardiac events (14-16). Accordingly, it was the objective of this study to assess the association of CAA with plasma glucose levels in asymptomatic, community-dwelling, Chinese, adults using DSCT as a proof-of-principle of the technology's screening value.

\section{Methods}

\section{Study population}

We recruited subjects for our study population from the permanent residents of the Songnan District of Shanghai. Potential subjects had to be 40 years of age or older without the prior diagnosis of cardiovascular disease. From a total of 4,200 volunteers, we selected every seventh subject for inclusion in this study based upon sequential registration. Of the 600 subjects initially selected, 263 were eliminated based upon the exclusion criteria listed in the supplemental material (Table S1). The remaining 337 participants were divided into three groups based upon their plasma glucose levels according to the 1999 World Health Organization criteria (17): (I) the DM group: fasting plasma glucose $(\mathrm{FPG}) \geq 7.0 \mathrm{mmol} / \mathrm{L}$, and $2-\mathrm{h}$ postprandial plasma glucose $(\mathrm{PPG}) \geq 11.1 \mathrm{mmol} / \mathrm{L}$; (II) the pre-diabetic group: $6.1 \mathrm{mmol} / \mathrm{L} \leq \mathrm{FPG}<7.0 \mathrm{mmol} / \mathrm{L}$ plus $\mathrm{PPG}<7.8 \mathrm{mmol} / \mathrm{L}$ or FPG $<7.0 \mathrm{mmol} / \mathrm{L}$ plus $7.8 \mathrm{mmol} / \mathrm{L} \leq \mathrm{PPG}<11.1 \mathrm{mmol} / \mathrm{L}$; and (III) the normal glucose regulation group (NGR): FPG $<6.1 \mathrm{mmol} / \mathrm{L}$, and PPG $<7.8 \mathrm{mmol} / \mathrm{L}$. The participants were further divided into two subsets, those with or without CAA, based upon their DSCT scan images. In this study, we focused on subjects with CAA to determine the relation between the prevalence, extent, and severity of CAA with plasma glucose parameters.

\section{Image protocol and analysis}

We performed plain and enhanced DSCT (Somatom Definition, Siemens Medical Solutions, Forchheim, Germany). After written informed consent was obtained from each participant, we conducted DSCT scanning in a craniocaudal direction covering the region from $1 \mathrm{~cm}$ caudal to the level of the tracheal bifurcation to the diaphragm. Scanning parameters were set before image acquisition was initiated.

Experienced cardiac imagers (Dr. Lingwei Yu and Dr. Ming Ding, two radiologists in our hospital who are also co-authors) selected the best image from each subject based upon image quality for quantitative analysis. The images were subjectively scored on a 4-point grading scale: perfect image quality $=4$; fully interpretable image quality $=3$; artifacts, but deemed interpretable $=2$; severe artifacts $=1$; uninterpretable $=0$. Images with quality scored of 1 or 0 were not included in the analysis. We analyzed each image according to a 16-segment model based upon the American Heart Association guidelines (18).

If a subject's scan showed the presence of an atherosclerotic plaque in any coronary artery segment, defined as a tissue structure $>1 \mathrm{~mm}^{2}$, we classified the subject as having CAA. We scored stenosis severity in each coronary artery segment based on the lumen reduction as follows: slight stenosis $(<25 \%)$, mild stenosis $(25-49 \%)$, 
moderate stenosis (50-74\%), or severe stenosis ( $\geq 75 \%)(18)$. Also, we identified plaques in each segment as calcified, non-calcified or mixed. We defined calcified plaques as plaques with high attenuation on CT compared with the contrasted lumen. We defined non-calcified plaques as any discernible structure in the coronary artery wall with a CT density less than the contrast-enhanced coronary lumen but higher than the surrounding connective tissue. We classified plaques that met these criteria but showed additional calcification as mixed plaques. Two experienced radiologists, who were blinded to all other data, analyzed the DSCT images for the severity of the stenosis. In case of a disagreement between the two examiners, a third experienced cardiologist mediated a consensus.

\section{Statistical analysis}

We analyzed data by using SPSS 16.0 (v16.0, IBM Corp., Armonk, NY, USA) software. We expressed quantitative variables as mean \pm 1 SD for normally distributed variables and medians with 25 th-75th percentiles for skewed, distributed variables. We used counts (\% of total) for categorical variables. We used chi-squared tests or Fisher's exact tests to compare the differences between selected groups for numeric and categorical variables, respectively. For continuous variables, we used two-tailed $t$-tests or Kruskal-Wallis rank sum tests. Demographic and metabolic features and characteristics of coronary arteries in the various groups were compared using ANOVA for continuous variables. We used logistic regression models adjusted for age, sex, smoking status, body mass index, SBP, family history of CAD, TC, TG, LDL-C, and HDL-C to determine the associations between CAA and diabetic parameters (glycemic status/HbA1c). A $\mathrm{P}<0.05$ was reported as statistically significant.

\section{Results}

\section{Study population and clinical characteristics}

We recruited a total of 337 subjects for this study of which 131 were in the NGR group, 174 in the pre-diabetic group, and 32 in the DM group. We excluded two subjects (one with diabetes and the other with NGR) because of a low DSCT image score. We divided the remaining 335 analyzed participants into two groups, with or without CAA, according to their DSCT images. Of the 335 participants, 118 were positive, and 217 were negative for CAA.
Table 1 summarizes the general characteristics of the 118 participants identified with CAA. There were no significant differences in age or gender distribution between the groups. Subjects with DM had significantly higher values of BMI, abdominal circumference, systolic blood pressure (SBP), diastolic blood pressure (DBP), TG, FPG, PPG, and $\mathrm{HbA1c}$ compared to subjects in the pre-diabetic or NGR groups. Values of all of these parameters except SBP, DBP, and HOMA-IR were significantly higher in the DM group compared to the pre-diabetic group. There was no difference in the percentage of smokers between the pre-diabetic group and the DM group while both were significantly larger than the NGR group.

\section{Distribution of stenosis severity and plaque type}

Typical DSCT images of coronary arteries from subjects in the three groups are shown in Figure 1. There was a positive trend for the percentage of subjects with CAA to increase with blood glucose level (Table 2): NGR $=27.7 \%$ $(36 / 130)$, pre-diabetes $=35.1 \%(61 / 174)$ and $\mathrm{DM}=67.7 \%$ $(21 / 31)$ (P value for trend $=0.031)$. One-vessel involvement was the most common pattern occurring in $60 \%(71 / 118)$ of the CAA positive subjects. Of the remaining subjects, $23.7 \%(28 / 118)$ had a two-segment disease, and $16.1 \%$ $(19 / 118)$ had three or more segment disease. Additionally, the number of segments with stenotic lesions increased with plasma glucose level from $8.3 \%$ in the NGR group to $12.7 \%$ in the pre-diabetic group to $20.2 \%$ in the DM group.

\section{Subclinical CAA and glucose metabolism}

We averaged plasma glucose parameters (FPG, PPG, and HbA1c) among the subjects at each level of coronary stenosis severity. There was a positive correlation between the severity of coronary stenosis and each plasma glucose parameter (Figure 2). Results from multivariable logistic regression models indicated a significantly higher risk of CAA among the diabetic subjects (Table 3). Among these subjects, the risk of CAA was more than 2-fold higher than for those in the NGR group [odds ratio $(\mathrm{OR})=2.23,95 \%$ CI: $1.87-4.98]$. CAA risk increased an average of $77 \%$ for each percent increment in HbA1c (OR $=1.98$, 95\% CI: 1.09-3.98). Multivariable logistic regression analysis (Table 3) also showed a significant association between high FPG, PPG, and HbA1c and an increased risk of CAA [adjusted OR (95\% CI): for pre-diabetes, 1.03 (0.36-2.25); 
Table 1 General clinical characteristics of subjects

\begin{tabular}{|c|c|c|c|c|}
\hline Variables & NGR & Pre-diabetes & DM & $P$ for trend \\
\hline Age (years) & $52.38 \pm 4.21$ & $52.72 \pm 4.24$ & $52.65 \pm 4.12$ & $>0.05$ \\
\hline Males (n, \%) & $20(55.6)$ & $33(54.1)$ & $12(57.1)$ & $>0.05$ \\
\hline Females (n, \%) & $16(44.4)$ & $28(45.9)$ & $9(42.9)$ & - \\
\hline Abdominal circumference $(\mathrm{cm})$ & $84.08 \pm 9.24$ & $87.06 \pm 8.65^{\star, \#}$ & $92.75 \pm 9.25^{\star}$ & $<0.001$ \\
\hline Systolic BP (mmHg) & $126.90 \pm 18.53$ & $138.17 \pm 20.37^{*}$ & $137.76 \pm 15.41^{*}$ & $<0.001$ \\
\hline Diastolic BP (mmHg) & $76.92 \pm 10.16$ & $81.44 \pm 9.91^{*, \#}$ & $83.86 \pm 8.08^{\star}$ & $<0.001$ \\
\hline Current smoker (n, \%) & $20(55.6)$ & $38(62.3)^{\S}$ & $13(61.9)^{\S}$ & $<0.05$ \\
\hline HDL cholesterol (mmol/L) & $1.37 \pm 0.30$ & $1.37 \pm 0.27$ & $1.33 \pm 0.30$ & $>0.05$ \\
\hline LDL cholesterol (mmol/L) & $2.30 \pm 0.65$ & $2.46 \pm 0.67$ & $2.60 \pm 0.65^{\S}$ & $>0.05$ \\
\hline $\mathrm{TG}(\mathrm{mmol} / \mathrm{L})$ & $1.58 \pm 1.08$ & $1.96 \pm 1.54^{*, \#}$ & $2.47 \pm 1.71^{*}$ & $<0.001$ \\
\hline $\mathrm{FPG}(\mathrm{mmol} / \mathrm{L})$ & $4.80 \pm 0.47$ & $6.11 \pm 1.02^{*, \#}$ & $9.20 \pm 2.88^{*}$ & $<0.001$ \\
\hline PPG (mmol/L) & $5.87 \pm 1.02$ & $9.97 \pm 2.37^{\star, \#}$ & $17.88 \pm 4.03^{*}$ & $<0.001$ \\
\hline $\mathrm{HbA1c}(\%)$ & $5.77 \pm 0.33$ & $6.31 \pm 0.66^{\star, \#}$ & $8.61 \pm 1.95^{*}$ & $<0.001$ \\
\hline
\end{tabular}

Values are means $\pm 1 \mathrm{SD} .{ }^{\S}, \mathrm{P}<0.05$ as compared to the NGR group; ${ }^{*}, \mathrm{P}<0.05$ versus the DM group; ${ }^{*}, \mathrm{P}<0.001$ compared to the NGR group. All comparisons are adjusted for age and gender. NGR, normal glucose regulation; DM, diabetes mellitus; BMI, body mass index; BP, blood pressure; CAD, coronary artery disease; TC, total cholesterol; HDL, high-density lipoprotein; LDL, low-density lipoprotein; TG, triglycerides; FPG, fasting plasma glucose; PPG, 2-hr postprandial plasma glucose; HbA1c, hemoglobin A1c.
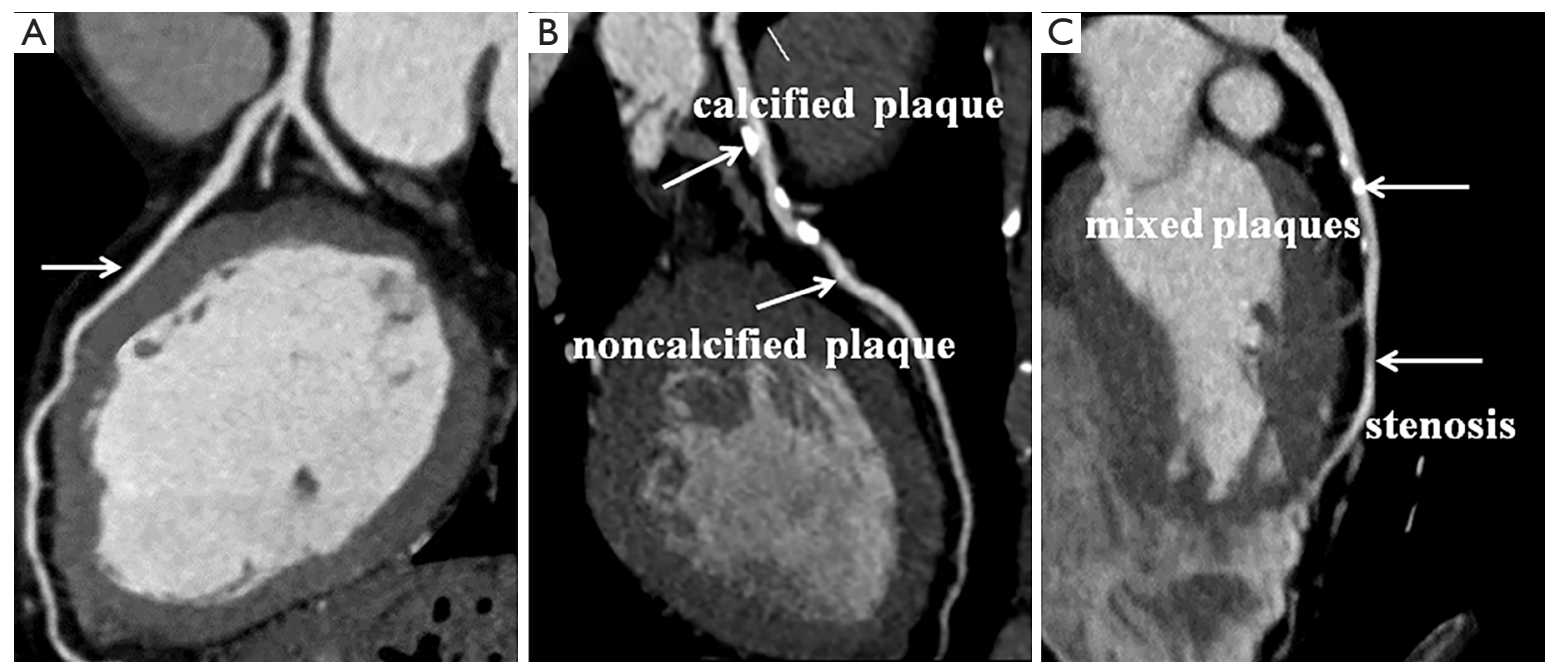

Figure 1 Representative coronary artery images obtained from subjects in each group using dual-source computed tomography. (A) Left coronary and left anterior descending arteries (arrow) from an NGR subject showing the absence of plaques and stenoses; (B) calcified and non-calcified plaques (arrows) in the coronary artery of a pre-diabetic subject; (C) mixed plaques and stenoses in a left anterior descending artery (arrows) in a diabetic subject. NGR, normal glucose regulation. 
Table 2 Distribution of severity and plaque type among subjects

\begin{tabular}{|c|c|c|c|c|c|}
\hline Parameter & Total & NGR & Pre-diabetes & DM & $P$ value \\
\hline Subjects with CAA, n (\%) & $118(35.2)$ & $36(27.7)$ & $61(35.1)$ & $21(67.7)$ & $\mathrm{P}<0.05$ \\
\hline Total segments, n (\%) & & 576 & 976 & 336 & \\
\hline Segments with CAA, $n(\%)$ & & $48(8.3)$ & $124(12.7)$ & $68(20.2)$ & $\mathrm{P}<0.05$ \\
\hline$<25 \%$ & & $39(81.2)$ & $2(1.6)$ & $1(1.5)$ & - \\
\hline $25-49 \%$ & & $9(18.8)$ & $46(37.1)$ & $18(26.5)$ & - \\
\hline $50-74 \%$ & & 0 & $7(5.6)$ & 7 (10.3) & - \\
\hline$>75 \%$ & & 0 & $69(55.7)$ & $42(61.7)$ & - \\
\hline Calcified plaques & & $9(1.7)$ & $24(2.5)$ & $33(9.8)$ & \\
\hline Non-calcified plaques & & $19(3.3)$ & $47(4.8)$ & $15(4.5)$ & \\
\hline Mixed plaques & & $20(3.5)$ & $53(5.4)$ & $20(6.0)$ & \\
\hline
\end{tabular}

NGR, normal glucose regulation; DM, diabetes mellitus; CAA, coronary artery atherosclerosis.

for DM, 2.11 (1.59-4.79); and for HbA1c, 1.77 (1.12-3.85)].

\section{Discussion}

In this study, we compared the prevalence and the severity of CAA, and its association with blood glucose parameters in asymptomatic community-dwelling subjects with no history of CAD using DSCT. We found 118 (35\%) of the 335 subjects in the study had CAA with an atherosclerotic lesion in at least one coronary artery segment. After dividing the subjects into groups based upon the accepted definitions of pre-diabetes and DM, the prevalence of CAA and the severity of coronary artery stenosis exhibited a positive correlation with blood glucose parameters, FPG, PPG, and HbAlc (P value for trend $=0.031$ ). Finally, the type of plaques present differed among the groups with calcified and mixed plaques predominating in the diabetic group versus mixed and non-calcified plaques predominating in the NGR group and the pre-diabetic group.

\section{Comparison to previous studies}

In general, our results are in agreement with most (19) but not with all (20) previous studies. For example, Park et al. (21) found that asymptomatic subjects with DM had more frequent subclinical CAA compared to matched subjects without DM, mostly confined to low-risk CAD. Gurudevan et al. (22) reported that asymptomatic individuals with impaired fasting glucose (IFG) and DM had significantly higher calcium scores, plaque burden, and obstructive CAD compared to those with IFG alone. In a study of 98 asymptomatic individuals with T2DM without known CAD, Rassi et al. (23) found a significant number (44\%) had CAD based upon CTA. In contrast, Binbrek et al. (24) found that only $5.5 \%$ of 1902 patients with T2DM in Dubai had normal coronary arteries. In the latter study, the group with normal coronary arteries had significantly lower LDL-C and higher HDL-C levels compared to the diseased group.

Two additional case-controlled, large observational studies showed an association between low HDL-C and increased cardiovascular risk $(25,26)$. Furthermore, subjects with dyslipidemia had significantly higher rates of cardiovascular events than in healthy subjects (27). MartínTimón et al. (28) also confirmed an association between dyslipidemia and cardiovascular risk in T2DM. In our study, although TG increased with blood glucose levels among the three groups and LDL-C was elevated in the DM group compared to the other two groups, we found no correlation between the severity of coronary artery 

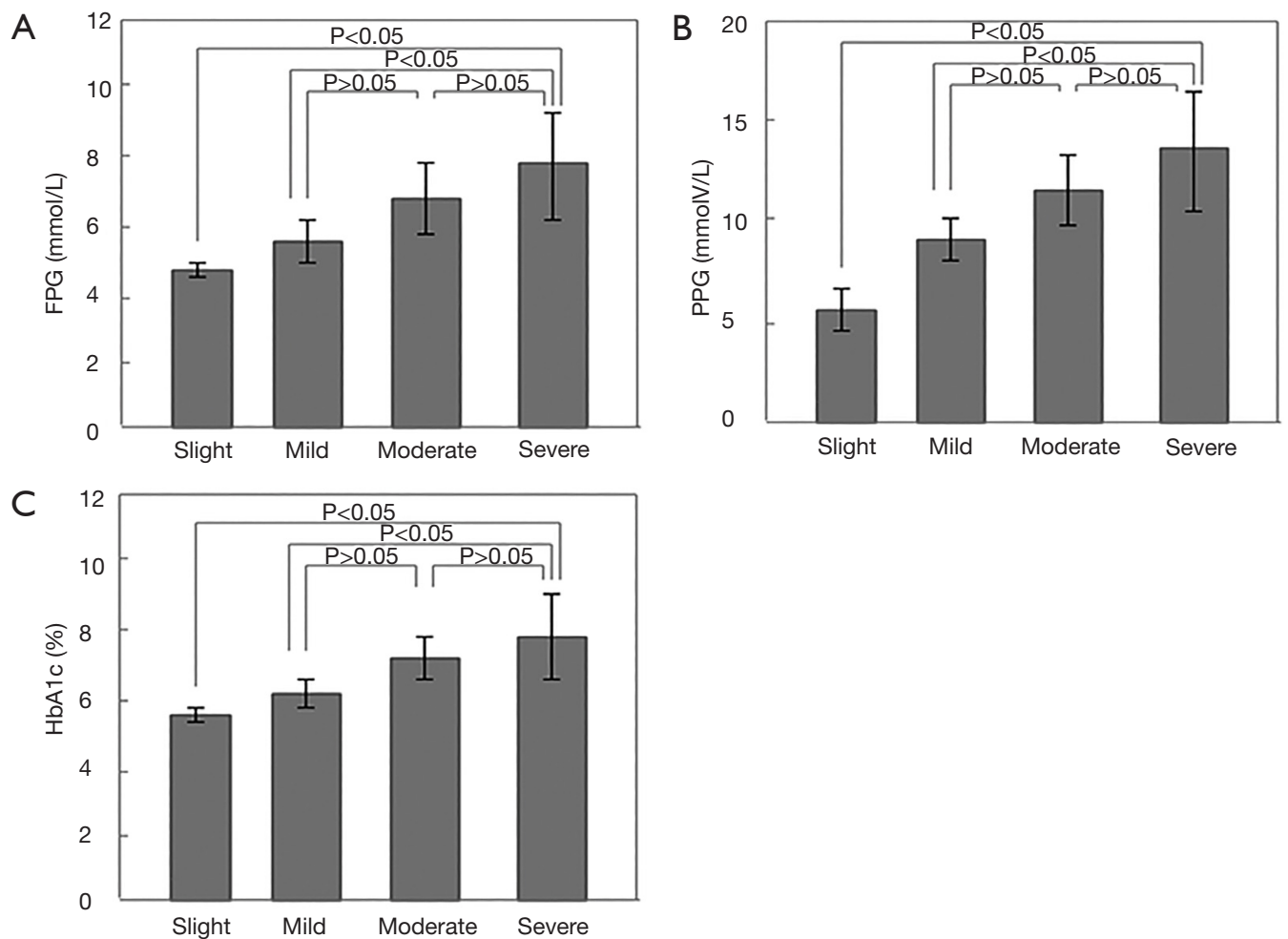

Figure 2 The relation between blood glucose parameters and the severity of coronary artery stenosis in all study subjects. Bars represent average levels of FPG, PPG, and HbA1C amongst participants versus the degree of coronary artery stenosis. T-bars indicate standard deviation. Degree of stenosis is defined as follows: slight ( $<25 \%$; $=42$ segments); mild (25-49\%; $n=73$ segments), moderate (50-74\%; $n=14$ segments) and severe ( $\geq 75 \% ; n=111$ segments). A: correlation with FPG; B: correlation of PPG; C: correlation with HbA1c. FPG, fasting plasma glucose; PPG, 2-hr postprandial plasma glucose; HbA1c, hemoglobin A1c.

Table 3 Odds ratios of coronary artery atherosclerosis (CAA) in pre-diabetic and diabetic participants compared to participants with normal glucose

\begin{tabular}{lcccc}
\hline \multirow{2}{*}{ Variable } & \multicolumn{2}{c}{ Crude } & & Adjusted \\
\cline { 2 - 3 } \cline { 5 - 5 } Glycemic status & ORs (95\% Cls) & P value & ORs (95\% Cls) & P value \\
NGR & 1.00 & $0.043^{*}$ & 1.00 & $0.031^{*}$ \\
Pre-diabetes & $1.18(0.52-2.16)$ & & $1.03(0.36-2.25)$ & 0.632 \\
Diabetes & $2.23(1.87-4.98)$ & 0.851 & $2.11(1.59-4.79)$ & 0.025 \\
HbA1c $(\%)$ & $1.98(1.09-3.98)$ & 0.031 & $1.77(1.12-3.85)$ & 0.013 \\
\hline
\end{tabular}

*, P value for trend for glycemic status (NGR, pre-diabetes, and diabetes). Odds ratios were calculated by using multiple logistic regression models. The following variables were adjusted in the model to calculate the adjusted OR, including age, sex, smoking status, body mass index, SBP, family history of CAD, TC, TG, LDL and HDL. NGR, normal glucose regulation; CAD, coronary artery disease; TC, total cholesterol; HDL, high-density lipoprotein; LDL, low-density lipoprotein; TG, triglycerides. 
stenosis and blood lipid parameters. We believe that may be related to an early pathological stage in our subjects where lipid concentrations may have changed, but no detectable CAD had developed. Notably, unlike our study, most other studies have been performed with subjects having confirmed $\mathrm{CAD}$ or suspected of having CAD.

Although numerous studies in symptomatic and asymptomatic populations have documented an association of DM with subclinical atherosclerosis $(19,29,30)$, there are limited data for pre-diabetic subjects that our study provides. Several previous cross-sectional studies have examined the association between fasting glucose levels and coronary artery calcium, and a crude association has been suggested similar to the present findings $(6,13,31)$. However, after multivariable adjustment to account for differences in patient mix, only some of the previously reported studies yielded a statistically significant association $(32,33)$. Xing et al. (34) evaluated the association of pre-diabetes with subclinical atherosclerosis in the Dallas Heart Study and found an apparent association of pre-diabetes with elevated coronary artery calcium that was not statistically significant after multivariable adjustments.

In our study, multivariate logistic regression analysis revealed a significantly increased occurrence of CAA in the DM group compared to the pre-diabetic and the NGR groups. Moreover, this analysis suggested that other risk factors for CAD might exist in pre-diabetes and diabetes that contributed to the elevated CAA prevalence. Notably, after multivariable adjustment to account for differences among the participants, the risk factors for coronary atherosclerosis in pre-diabetes and diabetes remained statistically significant, and they associated with a trend of an increase in CAA risk.

\section{Clinical significance}

In clinical practice, early detection of CAD is vital in order to initiate treatment early and prevent future adverse outcomes. Generally, non-calcified and mixed plaques are considered unstable and are likely to rupture leading to acute cardiac events (35). Such plaques were found to be more prevalent in the NGR and the pre-diabetes groups where combined they represented about $80 \%$ of the total plaque types. In contrast, in the diabetes group, these plaques represented about $52 \%$ of the total number. Our findings suggest that subjects in the NGR and the prediabetes groups may be at higher risk for future adverse events due to the higher prevalence of these potentially more dangerous plaque types.

To date, the prognostic value of coronary CT in patients with DM has not been thoroughly studied $(36,37)$. In a recent analysis of patients in the CONFIRM registry, Blanke et al. (38) reported that both nonobstructive and obstructive CAD in patients with DM were associated with higher rates of all-cause mortality and with major adverse cardiovascular events at five years compared to nondiabetic subjects. These results appear to be opposite to our predictions. There are several possible reasons for this apparent discrepancy. All subjects included in the CONFIRM registry had known or suspected CAD and underwent CTA by order of a physician (39). Compared to our study, subjects in the report of Blanke et al. (38) were older, of mixed ethnic backgrounds, studied at different participating sites, of various socioeconomic status, and subject to post-CTA treatments. Finally, they compared the degree of coronary occlusion in patients with recorded cardiovascular outcomes while our predictions were based on plaque type.

Regardless of the differences described above, our results suggest that the anatomic findings of CAA based upon DSCT may provide relevant prognostic information. These results highlight the importance of detecting CAA at its earliest stage by developing a risk stratification of patients especially those with pre-diabetes. Furthermore, we demonstrate that DSCT has the capability of detecting potentially more severe forms of CAA at an early stage when treatment can prevent serious adverse outcomes. Currently, we are following these patients in order to determine if DSCT results are predictive of a future cardiac event. We hope to report the results of these ongoing studies in the future.

\section{Limitations}

Despite the clinical implications of this study for CAD management in asymptomatic pre-diabetic subjects, it has some limitations. Firstly, the sample size was small which did not allow separate analyses by gender or cardiovascular risk stratification. Secondly, the presence of residual or undetected confounding cardiovascular risk factors could not be wholly ruled out. Thirdly, although HbA1c is also a criterion for the diagnosis of diabetes we did not use it in our analysis. Values of HbAlc in the three groups generally paralleled differences in FPG and PPG, and consequently do not provide additional insights. Also, HbA1c is not formally used as a diagnostic standard for diabetes in China 
because of the various methods used for HbAlc analysis, differences in the testing instruments, and the uneven quality of testing. Finally, the influence of some therapeutic drugs (such as statins) could not be assessed due to the small sample size of our study and the numbers of subjects on such drugs.

\section{Conclusions}

In summary, middle-aged, community-dwelling Chinese adults with different glycemic status underwent DSCT examination, which showed an increased prevalence and severity of both coronary plaques and CAD with increasing plasma glucose levels. We detected an increased risk of mild and moderate coronary stenosis in pre-diabetes without clinical manifestation of myocardial ischemia.

\section{Acknowledgements}

Funding: Funding for this study was provided in part by grant 15LC04 from the Clinical Research Unit Foundation of Xinhua Hospital affiliated with the School of Medicine (Dr. Yu); by grant 17411954800 from Shanghai Science and Technology Committee Clinical Field Project Fund (Dr. Yu): and by grant 201740136 from the Shanghai Health and Family Planning Commission (Dr. Yu).

\section{Footnote}

Conflicts of Interest: The authors have no conflicts of interest to declare.

Ethical Statement: The study was approved by Ethics Committee of Xin Hua Hospital Affiliated to Shanghai Jiao Tong University School of Medicine (No. XHEC-D-2011-027) and written informed consent was obtained from all patients.

\section{References}

1. International Diabetes Federation Atlas. About 415 million adults worldwide have diabetes. Medscape, December 02, 2015; Available online: http://www.diabetesatlas.org/. Accessed 5 July 2016.

2. Park SM, Miyazaki C, Prasad A, et al. Feasibility of prediction of myocardial viability with Doppler tissue imaging following percutaneous coronary intervention for ST-elevation anterior myocardial infarction. J Am Soc
Echocardiogr 2009;22:183-9.

3. Shemesh J, Henschke CI, Shaham D, et al. Ordinal scoring of coronary artery calcifications on low dose CT scans of the chest is predictive of death from cardiovascular disease. Radiology 2010;257:541-8.

4. Shemesh J, Motro M, Morag-Koren N, et al. Relation of coronary artery calcium to cardiovascular risk in patients with combined diabetes mellitus and systemic hypertension. Am J Cardiol 2012;109:844-50.

5. Rivera JJ, Nasir K, Choi EK, et al. Detection of occult coronary artery disease in asymptomatic individuals with diabetes mellitus using non-invasive cardiac angiography. Atherosclerosis 2009;203:442-8.

6. Yang HK, Kang B, Lee SH, et al. Association between hemoglobin A1c variability and subclinical coronary atherosclerosis in subjects with type 2 diabetes. J Diabetes Complicat 2015;29:776-82.

7. Kim JJ, Hwang BH, Choi IJ, et al. A prospective two-center study on the associations between microalbuminuria, coronary atherosclerosis and long-term clinical outcome in asymptomatic patients with type 2 diabetes mellitus: evaluation by coronary CT angiography. Int J Cardiovasc Imaging 2015;31:193-203.

8. Chow BJ, Wells GA, Chen L, et al. Prognostic value of 64-slice cardiac computed tomography severity of coronary artery disease, coronary atherosclerosis, and left ventricular ejection fraction. J Am Coll Cardiol 2010;55:1017-28.

9. Sun Z, Lin C, Davidson R, et al. Diagnostic value of 64-slice CT angiography in coronary artery disease: a systematic review. Eur J Radiol 2008;67:78-84.

10. Achenbach S, Ropers U, Kuettner A, et al. Randomized comparison of 64-slice single -and dual-source computed tomography coronary angiography for the detection of coronary artery disease. JACC Cardiovasc Imaging 2008;1:177-86.

11. Díaz-Zamudio M, Bacilio-Perez U, Herrera-Zarza MC, et al. Coronary artery aneurysms and ectasia, role of coronary CT angiography. Radiographics 2009;29:1939-54.

12. Ahmadi N, Nabavi V, Hajsadeghi F, et al. Mortality incidence of patients with non-obstructive coronary artery disease diagnosed by computed tomography angiography. Am J Cardiol 2011;107:10-6.

13. Chu ZG, Yang ZG, Dong ZH, et al. Characteristics of coronary artery disease in symptomatic type 2 diabetic patients: evaluation with CT angiography. Cardiovasc Diabetol 2010;9:1453-74.

14. Hadamitzky M, Meyer T, Hein F, et al. Prognostic value of coronary computed tomographic angiography in 
asymptomatic patients. Am J Cardiol 2010;105:1746-51.

15. Hadamitzky M, Freissmuth B, Meyer T, et al. Prognostic value of coronary computed tomographic angiography for prediction of cardiac events in patients with suspected coronary artery disease. JACC Cardiovasc Imaging 2009;2:404-11.

16. van Werkhoven JM, Schuijf JD, Gaemperli O, et al. Prognostic value of multislice computed tomography and gated single photon emission computed tomography in patients with suspected coronary artery disease. J Am Coll Cardiol 2009;53:623-32.

17. WHO consultation. Definition, diagnosis and classification of diabetes mellitus and it's complications. Part 1: diagnosis and classification of diabetes mellitus. 99.2. Geneva, World Health Organization. 1999.

18. Austen WG, Edwards JE, Frye RL, et al. A reporting system on patients evaluated for coronary artery disease. Report of the ad hoc committee for grading of coronary artery disease. Council on cardiovascular surgery, American Heart Association. Circulation 1975;51:5-40.

19. Scholte AJ, Schuijf JD, Kharagjitsingh AV, et al. Prevalence of coronary artery disease and plaque morphology assessed by multi-slice computed tomography coronary angiography and calcium scoring in asymptomatic patients with type 2 diabetes. Heart 2008;94:290-5.

20. Yun CH, Schlett CL, Rogers IS, et al. Association between diabetes and different components of coronary atherosclerotic plaque burden as measured by coronary multidetector computed tomography. Atherosclerosis 2009;205:481-5.

21. Park GM, Lee JH, Lee SW, et al. Comparison of coronary computed tomographic angiographic findings in asymptomatic subjects with versus without Diabetes Mellitus. Am J Cardiol 2015;116:372-8.

22. Gurudevan S, Garg P, Malik S, et al. Impaired fasting glucose is associated with increased severity of subclinical coronary artery disease compared to patients with diabetes and normal fasting glucose: evaluation by coronary computed tomographic angiography. BMJ Open 2016;6:e005148.

23. Rassi CH, Churchill TW, Tavares CA, et al. Use of imaging and clinical data to screen for cardiovascular disease in asymptomatic diabetics. Cardiovasc Diabetol 2016;15:28.

24. Binbrek AS, Ali SM, Baslaib FO, et al. Characteristics of patients with diabetes having normal coronary arteries. Angiology 2015;66:578-83.

25. Chapman MJ, Ginsberg HN, Amarenco P, et al.
Triglyceride-rich lipoproteins and high-density lipoprotein cholesterol in patients at high risk of cardiovascular disease: evidence and guidance for management. Eur Heart J 2011;32:1345-61.

26. Miller M, Stone NJ, Ballantyne C, et al. Triglycerides and cardiovascular disease: a scientific statement from the American Heart Association. Circulation 2011;123:2292-333.

27. Baigent C, Blackwell L, Emberson J, et al. Efficacy and safety of more intensive lowering of LDL cholesterol: a meta-analysis of data from 170,000 participants in 26 randomised trials. Lancet 2010;376:1670-81.

28. Martín-Timón I, Sevillano-Collantes C, Segura-Galindo A, et al. Type 2 diabetes and cardiovascular disease: Have all risk factors the same strength? World J Diabetes 2014;5:444-70.

29. Elkeles RS, Godsland IF, Feher MD, et al. Coronary calcium measurement improves prediction of cardiovascular events in asymptomatic patients with type 2 diabetes: the PREDICT study. Eur Heart J 2008;29:2244-51.

30. Malik S, Budoff MJ, Katz R, et al. Impact of subclinical atherosclerosis on cardiovascular disease events in individuals with metabolic syndrome and diabetes: the multi-ethnic study of atherosclerosis. Diabetes Care 2011;34:2285-90.

31. Moebus S, Stang A, Möhlenkamp S, et al. Association of impaired fasting glucose and coronary artery calcification as a marker of subclinical atherosclerosis in a populationbased cohort-results of the Heinz Nixdorf Recall study. Diabetologia 2009;52:81-9.

32. Iijima R, Ndrepepa G, Kujath V, et al. A pan-coronary artery angiographic study of the association between diabetes mellitus and progression or regression of coronary atherosclerosis. Heart Vessels 2017;32:376-84.

33. Kurihara O, Takano M, Seino Y, et al. Coronary atherosclerosis is already ongoing in pre-diabetic status: Insight from intravascular imaging modalities. World J Diabetes 2015;6:184-91.

34. Xing FY, Neeland IJ, Gore MO, et al. Association of prediabetes by fasting glucose and/or haemoglobinA1c levels with subclinical atherosclerosis and impaired renal function: Observations from the Dallas Heart Study. Diab Vasc Dis Res 2014;11:11-8.

35. Kitagawa T, Yamamoto H, Horiguchi J, et al. Characterization of noncalcified coronary plaques and identification of culprit lesions in patients with acute coronary syndrome by 64-slice computed tomography. 
JACC Cardiovasc Imaging 2009;2:153-60.

36. Andreini D, Pontone G, Mushtaq S, et al. Prognostic value of multidetector computed tomography coronary angiography in diabetes: excellent long-term prognosis in patients with normal coronary arteries. Diabetes Care 2013;36:1834-41.

37. Kim JJ, Hwang BH, Choi IJ, et al. Impact of diabetes duration on the extent and severity of coronary atheroma burden and long-term clinical outcome in asymptomatic type 2 diabetic patients: evaluation by coronary

Cite this article as: Yu Y, Zhou Z, Sun K, Xi L, Zhang L, Yu L, Wang J, Zheng J, Ding M. Association between coronary artery atherosclerosis and plasma glucose levels assessed by dualsource computed tomography. J Thorac Dis 2018;10(11):60506059. doi: $10.21037 /$ jtd.2018.10.62
CT angiography. Eur Heart J Cardiovasc Imaging 2015;16:1065-73.

38. Blanke P, Naoum C, Ahmadi A, et al. Long-term prognostic utility of coronary CT angiography in stable patients with Diabetes Mellitus. JACC Cardiovasc Imaging 2016;9:1280-8.

39. Min JK, Dunning A, Lin FY, et al. Rationale and design of the CONFIRM (Coronary CT Angiography Evaluation For Clinical Outcomes: An International Multicenter Registry). J Cardiovasc Comput Tomogr 2011;5:84-92. 
Supplementary

Table S1 Exclusion criteria

Exclusion criteria

Any acute symptoms of CAD (i.e., chest pain or shortness of breath)

History of cardiovascular disease (including myocardial infarction, unstable angina, percutaneous coronary intervention or stroke)

Unstable sinus rhythm

Previous experience of a life-threatening event

Diagnosis of diabetes in the past five years

Impaired liver or renal function with alanine aminotransferase $>$ twice the upper limit of the normal range, serum creatinine level $>133 \mathrm{mmol} / \mathrm{L}$ (1.5 mg/dL), or glomerular filtration rate $<60 \mathrm{~mL} / \mathrm{min}$

Pregnant or having significant medical comorbidities

X-ray examination or CT scan within one year

History of allergic reaction to iodine-containing contrast agent

CAD, coronary artery disease. 